\title{
Majorization, 4G Theorem and Schrödinger perturbations
}

\author{
KRZYSZTOF BOGDAN, YANA BUTKO AND KAROL SZCZYPKOWSKI
}

\begin{abstract}
Schrödinger perturbations of transition densities by singular potentials may fail to be comparable with the original transition density. For instance, this is so for the transition density of a subordinator perturbed by any time-independent unbounded potential. In order to estimate such perturbations, it is convenient to use an auxiliary transition density as a majorant and the $4 \mathrm{G}$ inequality for the original transition density and the majorant. We prove the $4 \mathrm{G}$ inequality for the $1 / 2$-stable and inverse Gaussian subordinators, discuss the corresponding class of admissible potentials and indicate estimates for the resulting transition densities of Schrödinger operators. The connection of the transition densities to their generators is made via the weak-type notion of fundamental solution.
\end{abstract}

\section{Introduction and preliminaries}

Schrödinger perturbation consists of adding to a given operator an operator of multiplication by a function $q$. On the level of inverse operators the addition results in the perturbation series. We focus on transition densities $p$ perturbed by functions $q \geq 0$. Our main goal is to give pointwise estimates for the resulting perturbation series $\tilde{p}$ under suitable integral conditions on $p$ and $q$. For instance, bounded potentials $q$ produce transition densities $\tilde{p}$ comparable with the original $p$ in finite time. In a series of recent papers, integral conditions leading to comparability of $\tilde{p}$ and $p$ were proposed which allow for rather singular potentials $q$, if $p$ satisfies the 3G Theorem $[2,4]$. The integral conditions compare the second term in the perturbation series (that which is linear in $q$ ) with $p$ (the first term of the series). The comparison is meant to prevent the instantaneous blowup and to control the long-time accumulation of mass. The first property may be secured by smallness conditions, like $0 \leq \eta<1$ below, and the second is accomplished by using a subadditive function $Q$. The results render $p$ an approximate majorant for $\tilde{p}$ in finite time [4]. They may also be considered as analogues of the Gronwall inequality [3]. We note that similar estimates for Green-type kernels were recently obtained in $[10,11,13]$.

The 3G Theorem, which is related to the quasi-metric condition [10], is common for transition densities with power-type decay, e.g., the transition density of the fractional Laplacian. However, many transition densities fail to satisfy $3 \mathrm{G}$, for instance, the Gaussian kernel. In [5] and [3], a more flexible majorization technique is proposed,

Mathematics Subject Classification: Primary 47D06, 47D08; Secondary 35A08, 35B25

Keywords: 4G inequality, Schrödinger perturbation, Subordinator, Fundamental solution. 
motivated by earlier results of [19]. Namely, another transition density $p^{*}$ serves as an approximate majorant for the perturbation series. Introducing $p^{*}$ is not merely a technical device: For unbounded $q, \tilde{p}$ may fail to be comparable with $p$ in finite time. Finding an appropriate $p^{*}$ is essentially tantamount to estimating $\tilde{p}$, cf. (1.12), and may be hard, but in some cases, including ours, it is sufficient to chose $p^{*}$ being a dilation of $p$. The $p^{*}$ majorization technique further involves an integral smallness condition for $p, q$ and $p^{*}$, which is implied by the familiar Kato-type conditions if $p$ and $p^{*}$ satisfy the $4 \mathrm{G}$ inequality.

In this paper, we prove a $4 \mathrm{G}$ inequality for the transition density $p$ of the inverse Gaussian subordinator, including the 1/2-stable subordinator. We reveal a wide class of unbounded Schrödinger potentials admissible for this $p$ and estimate the Schrödinger perturbations series for $p$ using the framework of [5]. We thus extend the scope of the $p^{*}$ majorization technique for Schrödinger perturbations, beyond the transition densities of diffusion processes discussed in [5]. We expect $4 \mathrm{G}$ to be valid quite generally, but at present it is even open for the $\alpha$-stable subordinators with $\alpha \neq 1 / 2$. We note that the methods of [4], which make assumptions on potentials $q$ in terms of bridges (see also [2]), fail for unbounded $q$ in this case. Namely, if $p$ is the transition density of a subordinator and $q$ is time independent and unbounded, then $p$ and $\tilde{p}$ are never comparable, as proved in Sect. 3. The results explain why we propose 4G and the framework of [5] as a viable general approach to Schrödinger perturbations of transition densities by unbounded functions $q$.

The structure of the paper is as follows. Below, in this section, we give notation and preliminaries. In Sect. 2, we present $4 \mathrm{G}$ inequality and applications to Kato-type perturbations for the 1/2-stable subordinator and the inverse Gaussian subordinator. In Sect. 3, we discuss unbounded perturbations $q$ of general subordinators. In Lemma 2.1 and in Sect. 4, we discuss the connection of the considered integral operators to generators, with focus on Lévy-type generators. In Remark 4.8, we indicate extensions of our results to the case of signed $q$.

Let $X$ be an arbitrary set with a $\sigma$-algebra $\mathcal{M}$ and a (nonnegative) $\sigma$-finite measure $m$ defined on $\mathcal{M}$. To simplify the notation, we write $\mathrm{d} z$ for $m(\mathrm{~d} z)$ in what follows. We also consider the $\sigma$-algebra $\mathcal{B}$ of Borel subsets of $\mathbb{R}$, and the Lebesgue measure, $\mathrm{d} u$, defined on $\mathbb{R}$. The space time, $\mathbb{R} \times X$, is equipped with the $\sigma$-algebra $\mathcal{B} \times \mathcal{M}$ and the product measure $\mathrm{d} u \mathrm{~d} z=\mathrm{d} u m(\mathrm{~d} z)$. We consider a measurable transition density $p$ on space time, i.e., we assume that $p: \mathbb{R} \times X \times \mathbb{R} \times X \rightarrow[0, \infty]$ is $\mathcal{B} \times \mathcal{M} \times \mathcal{B} \times \mathcal{M}$ measurable and the following Chapman-Kolmogorov equations hold for all $x, y \in X$ and $s<u<t$ :

$$
\int_{X} p(s, x, u, z) p(u, z, t, y) \mathrm{d} z=p(s, x, t, y) .
$$

All the functions considered below are assumed measurable on their respective domains. We consider a (nonnegative and $\mathcal{B} \times \mathcal{M}$-measurable) function $q: \mathbb{R} \times X \rightarrow$ $[0, \infty]$. The Schrödinger perturbation $\tilde{p}$ of $p$ by $q$ is defined as 


$$
\tilde{p}(s, x, t, y)=\sum_{n=0}^{\infty} p_{n}(s, x, t, y)
$$

where $p_{0}(s, x, t, y)=p(s, x, t, y)$ and, for $n=1,2, \ldots$,

$$
p_{n}(s, x, t, y)=\int_{s}^{t} \int_{X} p(s, x, u, z) q(u, z) p_{n-1}(u, z, t, y) \mathrm{d} z \mathrm{~d} u .
$$

The above is an explicit method of constructing new transition densities. In particular, $\tilde{p}$ satisfies the Chapman-Kolmogorov equations [2, Lemma 2]. From (1.3) and the perturbation series (1.2), we get the perturbation formula:

$$
\tilde{p}(s, x, t, y)=p(s, x, t, y)+\int_{s}^{t} \int_{X} p(s, x, u, z) q(u, z) \tilde{p}(u, z, t, y) \mathrm{d} z \mathrm{~d} u .
$$

We similarly get the following variant,

$$
\tilde{p}(s, x, t, y)=p(s, x, t, y)+\int_{s}^{t} \int_{X} \tilde{p}(s, x, u, z) q(u, z) p(u, z, t, y) \mathrm{d} z \mathrm{~d} u .
$$

Since $q \geq 0$, we trivially have $\tilde{p} \geq p$, so we focus on the upper bounds for $\tilde{p}$. These may be obtained under suitable conditions on $p_{1}$. In [4] (see also [2,15] and [20, Lemma 3.1]), the authors assume that for all $s<t, x, y \in X$,

$$
\int_{s}^{t} \int_{X} p(s, x, u, z) q(u, z) p(u, z, t, y) \mathrm{d} z \mathrm{~d} u \leq[\eta+Q(s, t)] p(s, x, t, y),
$$

where $0 \leq \eta<\infty$ and $Q$ is superadditive: $0 \leq Q(s, u)+Q(u, t) \leq Q(s, t)$. The following estimates follow: for all $s<t, x, y \in X$,

$$
\tilde{p}(s, x, t, y) \leq p(s, x, t, y)\left(\frac{1}{1-\eta}\right)^{1+Q(s, t) / \eta},
$$

provided $0<\eta<1$, and for $\eta=0$, we even have

$$
\tilde{p}(s, x, t, y) \leq p(s, x, t, y) e^{Q(s, t)} .
$$

The condition (1.6) may be considered as property of relative boundedness of $q$, or Miyadera-type condition for bridges [2,16]. It is convenient to use (1.6), e.g., for the transition density of the isotropic $\alpha$-stable Lévy process with $\alpha \in(0,2)$, because the so-called $3 \mathrm{G}$ inequality holds in this case:

$$
p(s, x, u, z) \wedge p(u, z, t, y) \leq c p(s, x, t, y), \quad s<u<t, x, y, z \in \mathbb{R}^{d} .
$$

$3 \mathrm{G}$ simplifies the verification of (1.6) allowing for a simple description of the acceptable growth of $q$, cf. [2, Corollary 11], [4, Section 4]. In general, however, condition (1.6) may be troublesome. For instance, the transition density of the Brownian motion fails to satisfy $3 \mathrm{G}$ and (1.6) is difficult to characterize in a simpler way. Moreover, as 
we see below, for some transition densities, (1.6) holds for $q(u, z)=q(z)$ (i.e., timeindependent $q$ ) only if $q$ is bounded. This explains the need for modifications of [4]. The approach of [5] is based on the assumption that for all $s<t, x, y \in X$,

$$
\int_{s}^{t} \int_{X} p(s, x, u, z) q(u, z) p^{*}(u, z, t, y) \mathrm{d} z \mathrm{~d} u \leq[\eta+Q(s, t)] p^{*}(s, x, t, y) .
$$

Here it is furthermore assumed that $0 \leq \eta<\infty, Q(s, t)$ is superadditive, rightcontinuous in $s$ and left-continuous in $t$ (in short: regular superadditive), and $p^{*}$ is a majorizing transition density, i.e., there is a constant $C \geq 1$ such that for all $s<t$ and $x, y \in X$,

$$
p(s, x, t, y) \leq C p^{*}(s, x, t, y) .
$$

The above assumptions are abbreviated to $q \in \mathcal{N}\left(p, p^{*}, C, \eta, Q\right)$. By [5, Theorem 1.1], if $q \in \mathcal{N}\left(p, p^{*}, C, \eta, Q\right)$ with $\eta<1$, then for every $\varepsilon \in(0,1-\eta)$,

$$
\tilde{p}(s, x, t, y) \leq p^{*}(s, x, t, y)\left(\frac{C}{1-\eta-\varepsilon}\right)^{1+\frac{Q(s, t)}{\varepsilon}}, \quad s<t, x, y \in X .
$$

For instance, $p^{*}(s, x, t, y)=p(s / c, x, t / c, y)=c^{d} p(c s, c x, c t, c y)$ with $c \in(0,1)$ is convenient for the Gaussian kernel in $\mathbb{R}^{d}$ [5], and $Q(s, t)=\beta(t-s)$ with a constant $\beta \geq 0$ is a common choice. In this work, we use similar dilations to produce $p^{*}$.

In principle, (1.9) relaxes (1.6) and allows for more functions $q$. This is seen in [5] and again in Sect. 3 below, where we consider applications to transition densities of subordinators. We should note that the flexibility comes at the expense of the sharpness of the resulting estimate, as seen when comparing (1.7) and (1.8) with (1.11). Also, the methods of [5] and the present paper are restricted to transition densities, while the methods of [4] handle the more general so-called forward integral kernels. Last but not least, it may be cumbersome to point out $p^{*}$ suitable for $p$ and $q$, because this essentially requires guessing the rate of inflation of $\tilde{p}$. In this connection, we note that (1.5) trivially yields

$$
\int_{s}^{t} \int_{X} p(s, x, u, z) \eta q(u, z) \tilde{p}(u, z, t, y) \mathrm{d} z \mathrm{~d} u \leq \eta \tilde{p}(s, x, t, y) .
$$

Thus, for perturbations of $p$ by $\eta q \geq 0$ with $0 \leq \eta<1$, one may take $p^{*}=\tilde{p}$; hence, estimating $\tilde{p}$ and finding an appropriate majorant $p^{*}$ are closely related problems. Comparing to the approach of [4], we finally note that $p^{*}$ should reflect the growth patterns of $\tilde{p}$, which $p$ is not always able to do.

We say that $q$ satisfies the parabolic Kato condition for $p$ if

$$
\lim _{h \rightarrow 0^{+}} \sup _{s \in \mathbb{R}, x \in X} \int_{s}^{s+h} \int_{X} p(s, x, u, z) q(u, z) \mathrm{d} z \mathrm{~d} u=0,
$$

and

$$
\lim _{h \rightarrow 0^{+}} \sup _{t \in \mathbb{R}, y \in X} \int_{t-h}^{t} \int_{X} p(u, z, t, y) q(u, z) \mathrm{d} z \mathrm{~d} u=0,
$$


cf. [2, (29), (30)]. The relations between (1.13), (1.14), 3G and (1.6) are discussed in [2, Lemma 9 and Corollary 11] and [2, (40), (7) and Lemma 5]. Similar connections exist for (1.9), parabolic Kato conditions and 4G, but we leave the details to the interested reader (see also the proof of Proposition 2.4).

Of particular interest here is the special case of convolution semigroups of probability measures $\left\{p_{t}\right\}_{t \geq 0}$ on $X=\mathbb{R}^{d}$, which are defined by the generating (Lévy) triplets $(A, b, v)[17]$ and correspond to the generators

$$
\begin{aligned}
L f(x)= & \frac{1}{2} \sum_{j, k=1}^{d} A_{j, k} \frac{\partial^{2} f}{\partial x_{j} \partial x_{k}}(x)+\sum_{j=1}^{d} b_{i} \frac{\partial f}{\partial x_{j}}(x) \\
& +\int_{\mathbb{R}^{d}}\left(f(x+y)-f(x)-\sum_{j=1}^{d} y_{j} \frac{\partial f}{\partial x_{j}}(x) \mathbb{1}_{|y| \leq 1}(y)\right) v(\mathrm{~d} y) .
\end{aligned}
$$

Namely, we let $P_{t} f(x)=\int_{\mathbb{R}^{d}} f(z+x) p_{t}(\mathrm{~d} z), t \geq 0$ and recall that $\left(P_{t}\right)_{t \geq 0}$ form a strongly continuous semigroup on $\left(C_{0}\left(\mathbb{R}^{d}\right),\|\cdot\|_{\infty}\right)$, whose infinitesimal generator $L$ satisfies (1.15) for $f \in C_{0}^{2}\left(\mathbb{R}^{d}\right)$ and $x \in \mathbb{R}^{d}$. Furthermore, for all $s \in \mathbb{R}, x \in \mathbb{R}^{d}$ and $\phi \in C_{c}^{\infty}\left(\mathbb{R} \times \mathbb{R}^{d}\right)$ (smooth compactly supported functions on space time $\mathbb{R} \times \mathbb{R}^{d}$ ), we have

$$
\int_{s}^{\infty} \int_{\mathbb{R}^{d}}\left[\partial_{u} \phi(u, x+z)+L \phi(u, x+z)\right] p_{u-s}(\mathrm{~d} z) \mathrm{d} u=-\phi(s, x) .
$$

The identity is essentially a consequence of the fundamental theorem of calculus. It is proved in Sect. 4 in the generality of strongly continuous operator semigroups. We also provide a uniqueness result there. A special case of $L$ is the Weyl derivative of order $1 / 2$ on the real line:

$$
\partial^{1 / 2} f(x)=\pi^{-1 / 2} \int_{x}^{\infty} f^{\prime}(z)(z-x)^{-1 / 2} \mathrm{~d} z, \quad f \in C_{c}^{1}(\mathbb{R}) .
$$

We then have

$$
p_{t}(\mathrm{~d} z)=(4 \pi)^{-1 / 2} t z^{-3 / 2} \exp \left\{-t^{2} /(4 z)\right\} 1_{z>0} \mathrm{~d} z
$$

the distribution of the 1/2-stable subordinator [17] (also called the Lévy subordinator). More generally, we let $\lambda \geq 0, \delta>0, z \in \mathbb{R}, t>0$, and

$$
p(t, z)=(4 \pi)^{-1 / 2} \delta t z^{-3 / 2} \exp \left\{-\frac{(\delta t-2 \sqrt{\lambda} z)^{2}}{4 z}\right\} 1_{z>0} .
$$

We note that $p(t, z)$ is the density function of the distribution of the inverse Gaussian subordinator $\xi_{t}=\inf \left\{s>0: B_{s}+\sqrt{2 \lambda} s=t \delta / \sqrt{2}\right\}$, where $B$ is the standard one-dimensional Brownian motion, cf. [1, Example 1.3.21] and [7, Table 4.4]. Alternatively, $p$ may be obtained from the density function of the $1 / 2$-stable subordinator by 
the Esscher transform and time rescaling (see [17, Example 33.15] or [7, Sec. 4.4.2]). Accordingly, the Lévy measure $\mu$ of the inverse Gaussian subordinator is obtained by the exponential tilting of the Lévy measure $v$ of the $1 / 2$-stable subordinator, where

$$
\nu(\mathrm{d} y)=\frac{1}{2 \sqrt{\pi}} y^{-3 / 2} 1_{y>0} \mathrm{~d} y \quad \text { and } \quad \mu(\mathrm{d} y)=\delta e^{-\lambda y} \nu(\mathrm{d} y) .
$$

The generator corresponding to the inverse Gaussian subordinator is calculated for $f \in C_{c}^{1}(\mathbb{R})$ as

$$
L f(x)=\frac{\delta}{2 \sqrt{\pi}} \int_{x}^{\infty} f^{\prime}(z) \Gamma_{\lambda}(-1 / 2, z-x) \mathrm{d} z .
$$

Here $\Gamma_{\lambda}(a, z)=\int_{z}^{\infty} e^{-\lambda y} y^{a-1} \mathrm{~d} y$ for $\lambda, z>0, a \in \mathbb{R}$, is the incomplete gamma function. For the readers's convenience, we prove (1.17) and (1.20) in Sect. 4. Some further discussion can be found in [6]. We also note that the Laplace exponent of $\xi_{t}$ is $u \mapsto \delta(\sqrt{u+\lambda}-\sqrt{\lambda})$ (see, e.g., [1, Example 1.3.21], [17, Example 8.11 and 33.15]).

\section{4G inequality for the inverse Gaussian subordinator}

Our main goal is to give conditions for and discuss consequences of (1.11). Let $\lambda \geq 0$ and $\delta>0$. Using (1.19) we define

$$
p(s, x, t, y)=p(t-s, y-x),
$$

if $s<t$ and $x, y \in \mathbb{R}$, and we let $p=0$ otherwise. It is a transition density on $X=\mathbb{R}$ with respect to the Lebesgue measure. We observe that $3 \mathrm{G}$ inequality does not hold for $p$. Indeed, if $u-s=t-u=z-x=y-z=\theta$, then

$$
\begin{aligned}
p(s, x, u, z) \wedge p(u, z, t, y) & =(4 \pi)^{-1 / 2} \delta \theta^{-1 / 2} \exp \left\{-\theta(\delta-\sqrt{\lambda})^{2} / 4\right\}, \\
p(s, x, t, y) & =(4 \pi)^{-1 / 2} \delta(2 \theta)^{-1 / 2} \exp \left\{-2 \theta(\delta-\sqrt{\lambda})^{2} / 4\right\},
\end{aligned}
$$

and the second expression decays exponentially faster as $\theta \rightarrow \infty$. For $c>0$, we consider auxiliary (inverse Gaussian) transition density

$$
\rho_{c}(s, x, t, y):=c p(c(t-s), c(y-x)) .
$$

In view toward (1.10), we note that for $0<a<b$,

$$
\rho_{b}(s, x, t, y) \leq(b / a)^{1 / 2} \rho_{a}(s, x, t, y) .
$$

We shall consider the Schrödinger perturbation $\tilde{p}$ of $p=\rho_{1}$ by $q$. Clearly, if $q \in$ $\mathcal{N}\left(\rho_{1}, \rho_{a},(1 / a)^{1 / 2}, Q, \eta\right)$, with $0<a<1, \eta \in[0,1)$, then $\tilde{p}$ is finite; in fact, it satisfies (1.11). Here is a connection to generators. 
LEMMA 2.1. If $q \in \mathcal{N}\left(\rho_{1}, \rho_{a},(1 / a)^{1 / 2}, Q, \eta\right)$, where $0<a<1, \eta \in[0,1)$, then

$$
\int_{s}^{\infty} \int_{\mathbb{R}} \tilde{p}(s, x, u, z)\left[\partial_{u} \phi(u, z)+L \phi(u, z)+q(u, z) \phi(u, z)\right] \mathrm{d} z \mathrm{~d} u=-\phi(s, x),
$$

for $\phi \in C_{c}^{\infty}(\mathbb{R} \times \mathbb{R})$, where $L$ is given by $(1.20)$.

Proof. We will follow the proof of [4, Lemma 4] with some modifications. We define integral operators

$$
\begin{aligned}
P f(s, x) & =\int_{s}^{\infty} \int_{\mathbb{R}} p(s, x, t, y) f(t, y) \mathrm{d} y \mathrm{~d} t, \\
q f(s, x) & =q(s, x) f(s, x), \\
\tilde{P} f(s, x) & =\int_{s}^{\infty} \int_{\mathbb{R}} \tilde{p}(s, x, t, y) f(t, y) \mathrm{d} y \mathrm{~d} t, \\
P^{*} f(s, x) & =\int_{s}^{\infty} \int_{\mathbb{R}} \rho_{a}(s, x, t, y) f(t, y) \mathrm{d} y \mathrm{~d} t,
\end{aligned}
$$

for $s, x \in \mathbb{R}$ and jointly measurable and nonnegative or absolutely integrable functions $f: \mathbb{R} \times \mathbb{R} \rightarrow \mathbb{R}$. By (1.4) and (1.5),

$$
\tilde{P}=P+P q \tilde{P}=P+\tilde{P} q P
$$

hence, $\tilde{P} q P=P q \tilde{P}$. Let $\phi \in C_{c}^{\infty}(\mathbb{R} \times \mathbb{R})$ and $\psi=\partial_{s} \phi+L \phi$. By (1.16),

$$
\int_{s}^{\infty} \int_{\mathbb{R}} p(s, x, u, z)\left[\partial_{u} \phi(u, z)+L \phi(u, z)\right] \mathrm{d} z \mathrm{~d} u=-\phi(s, x) .
$$

In short, $P \psi=-\phi$. For clarity, since $\psi$ is bounded [17, p. 211] and $\psi(s, x)=0$ if $|s|$ is large, we have $P|\psi|<\infty$. By (2.3) and Fubini's theorem,

$$
\tilde{P}(\psi+q \phi)=(P+\tilde{P} q P) \psi+\tilde{P} q \phi=-\phi+\tilde{P} q P \psi+\tilde{P} q(-P \psi)=-\phi .
$$

The identity is precisely the claim of the lemma, but we need to verify the absolute convergence of the integrals above. Since $q \in \mathcal{N}\left(\rho_{1}, \rho_{a},(1 / a)^{1 / 2}, Q, \eta\right)$, we have $P q P^{*} \leq c P^{*}$ and $\tilde{P} \leq c P^{*}$, when applied to nonnegative functions in a bounded time horizon, cf. (1.9) and (1.11). It follows that in bounded time,

$$
\tilde{P} q P|\psi|=P q \tilde{P}|\psi| \leq c P q P^{*}|\psi| \leq c P^{*}|\psi|<\infty .
$$

Since $|\phi| \leq P|\psi|$, we get $\tilde{P} q|\phi|<\infty$. The proof is complete.

Following [8, Theorem 1.1] and [5], the identity in the statement of Lemma 2.1 is interpreted by saying that $\tilde{p}$ is a fundamental solution of $\partial_{s}+L+q$ or, in short, for $L+q$. The identity also means that $\tilde{p}$ as integral operator is the left inverse of $\partial_{s}+L+q$. We refer to [5, Remark 4.10] for further discussion. 
We point yet another aspect of the relationship between $\tilde{p}$ and $L+q$. By Lemma 2.1 and Chapman-Kolmogorov, for $\phi \in C_{c}^{\infty}(\mathbb{R} \times \mathbb{R})$, we obtain

$$
\begin{aligned}
\int_{s}^{t} & \int_{\mathbb{R}} \tilde{p}(s, x, u, z)\left[\partial_{u}+L+q(u, z)\right] \phi(u, z) \mathrm{d} z \mathrm{~d} u \\
= & \int_{s}^{\infty} \int_{\mathbb{R}} \tilde{p}(s, x, u, z)\left[\partial_{u}+L+q(u, z)\right] \phi(u, z) \mathrm{d} z \mathrm{~d} u \\
& -\int_{t}^{\infty} \int_{\mathbb{R}} \tilde{p}(s, x, u, z)\left[\partial_{u}+L+q(u, z)\right] \phi(u, z) \mathrm{d} z \mathrm{~d} u \\
= & -\phi(s, x)-\int_{\mathbb{R}} \tilde{p}(s, x, t, w) \int_{t}^{\infty} \int_{\mathbb{R}} \tilde{p}(t, w, u, z) \\
& \times\left[\partial_{u}+L+q(u, z)\right] \phi(u, z) \mathrm{d} z \mathrm{~d} u \mathrm{~d} w \\
= & -\phi(s, x)+\int_{\mathbb{R}} \tilde{p}(s, x, t, w)\left[-\int_{t}^{\infty} \int_{\mathbb{R}} \tilde{p}(t, w, u, z)\right. \\
& \left.\times\left[\partial_{u}+L+q(u, z)\right] \phi(u, z) \mathrm{d} z \mathrm{~d} u \mathrm{~d} w\right] \\
= & \int_{\mathbb{R}} \tilde{p}(s, x, t, w) \phi(t, w) \mathrm{d} w-\phi(s, x), \quad s<t, x \in \mathbb{R},
\end{aligned}
$$

and by choosing $\phi$ constant in time on $(s, t)$, for $\varphi \in C_{c}^{\infty}(\mathbb{R})$, we get

$$
\int_{\mathbb{R}} \tilde{p}(s, x, t, z) \varphi(z) \mathrm{d} z-\varphi(x)=\int_{s}^{t} \int_{\mathbb{R}} \tilde{p}(s, x, u, z)[L \varphi(z)+q(u, z) \varphi(z)] \mathrm{d} z \mathrm{~d} u .
$$

The identity is an analogue of classical formulas for strongly continuous operator semigroups, and so is (1.5). Further discussion of the connection to generators is given in Sect. 4.

We now investigate the class $\mathcal{N}\left(\rho_{b}, \rho_{a},(b / a)^{1 / 2}, \eta, Q\right)$, where $0<a<b$; namely, we propose conditions sufficient for (1.9). We first recall results of [5, Section 3] on the Gaussian kernel

$$
g_{c}(s, \bar{x}, t, \bar{y}):=[4 \pi(t-s) / c]^{-d / 2} \exp \left\{-|\bar{y}-\bar{x}|^{2} /[4(t-s) / c]\right\},
$$

where $c>0,0<s<t, \bar{x}, \bar{y} \in \mathbb{R}^{d}$ and $d \in \mathbb{N}$. We denote

$$
l(\alpha)=\max _{\tau \geq \alpha \vee 1 / \alpha}\left[\ln (1+\tau)-\frac{\tau-\alpha}{1+\tau} \ln (\alpha \tau)\right],
$$

and for $0<a<b$ we let $M=\left(\frac{b}{b-a}\right)^{d / 2} \exp \left[\frac{\mathrm{d}}{2} l\left(\frac{a}{b-a}\right)\right]$. Then, we have

$$
\frac{g_{b}(s, \bar{x}, u, \bar{z}) g_{a}(u, \bar{z}, t, \bar{y})}{g_{a}(s, \bar{x}, t, \bar{y})} \leq M\left[g_{b-a}(s, \bar{x}, u, \bar{z}) \vee g_{a}(u, \bar{z}, t, \bar{y})\right],
$$

where $s<u<t$ and $\bar{x}, \bar{z}, \bar{y} \in \mathbb{R}^{d}$ [5, Theorem 1.3 and Remark 3.2]. Moreover, $M$ is the optimal constant in (2.5), and if $b / a \leq 1+e^{-1 / 2}$, then $M=(1-a / b)^{-d}$. This 4G 
inequality is used in [5] to obtain Gaussian estimates for Schrödinger perturbations of transition densities of the second-order parabolic differential operators. In this section, we prove a similar inequality for the transition density $\rho_{c}$ defined in (2.1).

THEOREM 2.2 (4G). Let $0<a<b$. For all $s<u<t$ and $x<z<y$,

$\rho_{b}(s, x, u, z) \rho_{a}(u, z, t, y) \leq D\left[\rho_{b-a}(s, x, u, z) \vee \rho_{a}(u, z, t, y)\right] \rho_{a}(s, x, t, y)$

holds with $D=\left(\frac{b}{b-a}\right)^{3 / 2} \exp \left[\frac{3}{2} L\left(\frac{a}{b-a}\right)\right]$.

Proof. We denote $\bar{r}=(r, 0,0) \in \mathbb{R}^{3}$ for $r \in \mathbb{R}$. For $c>0, s<t, x<y$,

$$
\rho_{c}(s, x, t, y)=(4 \pi \delta(t-s) / c) g_{c}(x, \delta \bar{s}-2 \sqrt{\lambda} \bar{x}, y, \delta \bar{t}-2 \sqrt{\lambda} \bar{y}) .
$$

By (2.5) for all $s<u<t$ and $x<z<y$, we have

$$
\begin{aligned}
& \rho_{b}(s, x, u, z) \rho_{a}(u, z, t, y)=\frac{(4 \pi \delta)^{2}(u-s)(t-u)}{a b} \\
& \quad \times g_{b}(x, \delta \bar{s}-2 \sqrt{\lambda} \bar{x}, z, \delta \bar{u}-2 \sqrt{\lambda} \bar{z}) g_{a}(z, \delta \bar{u}-2 \sqrt{\lambda} \bar{z}, y, \delta \bar{t}-2 \sqrt{\lambda} \bar{y}) \\
& \leq \frac{(4 \pi \delta)^{2}(u-s)(t-u)}{a b} D g_{a}(x, \delta \bar{s}-2 \sqrt{\lambda} \bar{x}, y, \delta \bar{t}-2 \sqrt{\lambda} \bar{y}) \\
& \quad \times\left[g_{b-a}(x, \delta \bar{s}-2 \sqrt{\lambda} \bar{x}, z, \delta \bar{u}-2 \sqrt{\lambda} \bar{z}) \vee g_{a}(z, \delta \bar{u}-2 \sqrt{\lambda} \bar{z}, y, \delta \bar{t}-2 \sqrt{\lambda} \bar{y})\right] \\
&=D\left[\left(\frac{t-u}{t-s} \frac{b-a}{b}\right) \rho_{b-a}(s, x, u, z) \vee\left(\frac{u-s}{t-s} \frac{a}{b}\right) \rho_{a}(u, z, t, y)\right] \rho_{a}(s, x, t, y) \\
& \leq D\left[\rho_{b-a}(s, x, u, z) \vee \rho_{a}(u, z, t, y)\right] \rho_{a}(s, x, t, y) .
\end{aligned}
$$

We are ready to give sufficient conditions for (1.9). First comes an immediate consequence of Theorem 2.2.

COROLLARY 2.3. Assume that for all $s<t, x<y$,

$$
D \int_{s}^{t} \int_{\mathbb{R}}\left[\rho_{b-a}(s, x, u, z)+\rho_{a}(u, z, t, y)\right] q(u, z) \mathrm{d} z \mathrm{~d} u \leq \eta+Q(s, t) .
$$

Then, $q \in \mathcal{N}\left(\rho_{b}, \rho_{a},(b / a)^{1 / 2}, \eta, Q\right)$.

Motivated by (1.13) and (1.14) for $c, h>0$, we next define

$$
\begin{aligned}
N_{h}^{c}(q)= & \sup _{s, x} \int_{s}^{s+h} \int_{\mathbb{R}} \rho_{c}(s, x, u, z) q(u, z) \mathrm{d} z \mathrm{~d} u \\
& +\sup _{t, y} \int_{t-h}^{t} \int_{\mathbb{R}} \rho_{c}(u, z, t, y) q(u, z) \mathrm{d} z \mathrm{~d} u .
\end{aligned}
$$


PROPOSITION 2.4. Let $0<a<b$ and $D^{\prime}=\left(\frac{b-a}{a} \vee \frac{a}{b-a}\right)^{1 / 2}$ D. If

$$
N_{h}^{(b-a) \wedge a}(q) \leq \eta / D^{\prime}
$$

for some $0<h \leq \infty, 0 \leq \eta<\infty$, then for $Q(s, t)=\eta(t-s) / h$ we have

$$
q \in \mathcal{N}\left(\rho_{b}, \rho_{a},(b / a)^{1 / 2}, \eta, Q\right) .
$$

Proof. Follow [5, p. 165].

The condition $\lim _{h \rightarrow 0} N_{h}^{c}(q)=0$ defines the parabolic Kato class for $\rho_{c}$, cf. Sect. 1, and if it is satisfied, then Proposition 2.4 applies. A thorough discussion of the Kato condition for arbitrary Lévy processes on $\mathbb{R}^{d}$ is given in [12]. For the considered inverse Gaussian subordinator (1.19), including the 1/2-stable subordinator, if $q(u, z)=q(z)$ is time independent, then the Kato condition is equivalent to

$$
\lim _{r \rightarrow 0^{+}} \sup _{x \in \mathbb{R}} \int_{x-r}^{x+r} q(z)|z-x|^{-1 / 2} \mathrm{~d} z=0 .
$$

We refer to [12, Example 3] for the result. A characteristic example here is $q(z)=$ $|z|^{\varepsilon-1 / 2}$ for $\varepsilon \in(0,1 / 2]$.

In the remainder of this section, we focus on the case $\lambda=0$ and $\delta=1$ in (1.19), i.e., on the density of the $1 / 2$-stable subordinator, with emphasis on honest constants in estimates.

EXAMPLE. We consider $q(u, z)=q(z)$ on $\mathbb{R}$. Let $r>2$ and $q \in L^{r}(\mathbb{R})$. Observe that for all $s<u, x \in \mathbb{R}$ and $c>0$,

$$
\int_{\mathbb{R}} \rho_{c}(s, x, u, z)^{\sigma} \mathrm{d} z=\frac{c_{\sigma}^{\prime}}{c^{\sigma-1}}(u-s)^{-2(\sigma-1)}, \quad \sigma \geq 1,
$$

where $c_{\sigma}^{\prime}=(4 \pi)^{-\sigma / 2}(4 / \sigma)^{3 \sigma / 2-1} \Gamma(3 \sigma / 2-1) \leq\left[(4 \pi)^{-1 / 2}(6 / e)^{3 / 2}\right]^{\sigma-1}$. By Hölder's inequality, for $h>0$,

$$
\begin{aligned}
\sup _{s, x} & \int_{s}^{s+h} \int_{\mathbb{R}} \rho_{c}(s, x, u, z) q(z) \mathrm{d} z \mathrm{~d} u \\
& \leq \sup _{s, x} \int_{s}^{s+h}(u-s)^{-2 / r} \mathrm{~d} u \frac{\left(c_{r /(r-1)}^{\prime}\right)^{(r-1) / r}}{c^{1 / r}}\|q\|_{r} \\
& =h^{1-2 / r}\left[\left(c_{r /(r-1)}^{\prime}\right)^{(r-1) / r} c^{-1 / r}\|q\|_{r} /(1-2 / r)\right] .
\end{aligned}
$$

Thus, for every $c>0$,

$$
N_{h}^{c}(q) \leq h^{1-2 / r} 2\left[\frac{\left(c_{r /(r-1)}^{\prime}\right)^{(r-1) / r}}{(1-2 / r) c^{1 / r}}\|q\|_{r}\right] \rightarrow 0, \quad \text { if } h \rightarrow 0^{+} .
$$


Notice also that $\left(c_{r /(r-1)}^{\prime}\right)^{(r-1) / r} \leq\left[(4 \pi)^{-1 / 2}(6 / e)^{3 / 2}\right]^{1 / r}$. Finally, by Proposition 2.4 for all $0<a<b$, we obtain

$$
q \in \mathcal{N}\left(\rho_{b}, \rho_{a},(b / a)^{1 / 2}, \eta, Q\right),
$$

with arbitrary $\eta>0$ and $Q(s, t)=\eta(t-s) / h$, provided $h$ satisfies

$$
h^{1-2 / r} \frac{2 D\left(\frac{b-a}{a} \vee \frac{a}{b-a}\right)^{1 / 2}}{(1-2 / r)}\left[\frac{(4 \pi)^{-1 / 2}(6 / e)^{3 / 2}}{(b-a) \wedge a}\right]^{1 / r}\|q\|_{r}=\eta .
$$

Indeed, (2.8) implies (2.7).

We keep investigating the class $\mathcal{N}\left(\rho_{b}, \rho_{a},(b / a)^{1 / 2}, \eta, Q\right)$ by estimating $N_{h}^{c}(q)$ for time-independent $q(u, z)=q(z)$. We first prove an auxiliary lemma for the general $\alpha$-stable subordinator, with $\alpha \in(0,1)$. Let

$$
I_{\varepsilon}(q)=\sup _{x \in \mathbb{R}} \int_{|x-z|<\varepsilon} \frac{q(z)}{|x-z|^{1-\alpha}} \mathrm{d} z, \quad \varepsilon>0 .
$$

Let $\gamma(t, z)$ be the density of the $\alpha$-stable subordinator, in particular, $\gamma(t, z)=0$ for $z \leq 0$ and

$$
\int_{0}^{\infty} e^{-u z} \gamma(t, z) \mathrm{d} z=e^{-t u^{\alpha}}, \quad u \geq 0, \quad t>0 .
$$

LEMMA 2.5. For all $c, r, \tau>0$ and $0<\alpha<1$,

$$
\sup _{s \in \mathbb{R}, x \in \mathbb{R}} \int_{s}^{s+\tau} \int_{\mathbb{R}} \gamma_{c}(s, x, u, z) q(z) \mathrm{d} z \mathrm{~d} u \leq\left(\frac{1}{c^{1-\alpha} \Gamma(\alpha)}+\frac{2 \tau}{r^{\alpha}}\right) I_{r}(q),
$$

where $\gamma_{c}(s, x, t, y)=c \gamma(c(t-s), c(y-x))=\gamma\left(c^{1-\alpha}(t-s), y-x\right)$.

Proof. Let $c>0, k(x)=\int_{0}^{\tau} \gamma_{c}(0,0, u,|x|) \mathrm{d} u, K(x)=\int_{0}^{\infty} \gamma_{c}(0,0, u,|x|) \mathrm{d} u=$ $|x|^{\alpha-1} /\left(c^{1-\alpha} \Gamma(\alpha)\right), c_{1}=\int_{\mathbb{R}} k(x) d x=2 \tau$ and $c_{2}=r K(r)=r^{\alpha} /\left(c^{1-\alpha} \Gamma(\alpha)\right)$. By scaling, $\gamma_{c}(0,0, u,|x|)=|x|^{-1} \gamma_{c}\left(0,0,|x|^{-\alpha} u, 1\right)$. By a change in variables, $k$ is symmetrically decreasing. The result then follows from [5, Lemma 4.2].

A direct consequence is that for every $\alpha$-stable subordinator and for all $s<t, x<y$ and $h>0$, we have

$$
\begin{aligned}
\int_{s}^{t} \int_{\mathbb{R}} & {\left[\gamma_{b-a}(s, x, u, z)+\gamma_{a}(u, z, t, y)\right] q(z) \mathrm{d} z \mathrm{~d} u } \\
& \leq I_{h^{1 / \alpha}}(q)\left[\frac{1}{\Gamma(\alpha)} \frac{a^{1-\alpha}+(b-a)^{1-\alpha}}{[a(b-a)]^{1-\alpha}}+\frac{4(t-s)}{h}\right] .
\end{aligned}
$$

For $\alpha=1 / 2$, we may use Theorem 2.2 to get for all $s<t, x<y$ and $h>0$,

$$
\begin{aligned}
& \int_{s}^{t} \int_{\mathbb{R}} \rho_{b}(s, x, u, z) q(z) \rho_{a}(u, z, t, y) \mathrm{d} z \mathrm{~d} u \\
& \quad \leq D I_{h^{2}}(q)\left[\frac{1}{\Gamma(1 / 2)} \frac{\sqrt{a}+\sqrt{b-a}}{\sqrt{a(b-a)}}+\frac{4(t-s)}{h}\right] \rho_{a}(s, x, t, y) .
\end{aligned}
$$


COROLLARY 2.6. Let $q: \mathbb{R} \rightarrow \mathbb{R}$ be such that $I_{h^{2}}(q)<\infty$ for some $h>0$. Then, $q \in \mathcal{N}\left(\rho_{b}, \rho_{a},(b / a)^{1 / 2}, \eta, Q\right)$ with

$$
\begin{aligned}
& \eta=D I_{h^{2}}(q)(\sqrt{a}+\sqrt{b-a}) /(\Gamma(1 / 2) \sqrt{a(b-a)}), \\
& Q(s, t)=4 D I_{h^{2}}(q)(t-s) / h .
\end{aligned}
$$

Summarizing this section, we see that $4 \mathrm{G}$ for the inverse Gaussian subordinator yields (1.9) for a large class of functions $q$ characterized by simpler Kato-type conditions, and then, $\tilde{p}$ satisfies (1.11) and Lemma 2.1 .

\section{Relative boundedness for subordinators with transition density}

In this section, we consider a general subordinator with transition density $p$. Thus, $p$ is space time homogeneous, $p(s, x, t, y)=0$ whenever $t \leq s$ or $y \leq x$, and $p(s, x, t, y)>0$ otherwise. We first discuss time-independent functions $q$, aiming at the condition (1.6).

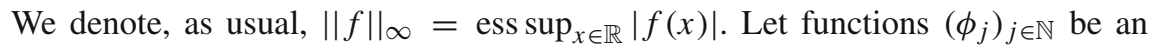
approximation to identity in $L^{1}(\mathbb{R})$, that is real-valued on $\mathbb{R}$ with the following properties:

$$
\begin{aligned}
& \phi_{j} \geq 0 \text { and } \int_{\mathbb{R}} \phi_{j}(z) \mathrm{d} z=1, \\
& \forall_{\delta>0} \exists_{j_{0} \in \mathbb{N}^{\forall}}{ }_{j \geq j_{0}} \operatorname{supp}\left(\phi_{j}\right) \subset(-\delta, \delta) .
\end{aligned}
$$

LEMMA 3.1. Let $f \in L_{\text {loc }}^{1}(\mathbb{R})$. If $\sup _{n \in \mathbb{N}}\left\|\phi_{n} * f\right\|_{\infty}<\infty$, then $f \in L^{\infty}(\mathbb{R})$.

Proof. Let $0<\delta<R$ and $M=\sup _{n \in \mathbb{N}}\left\|\phi_{n} * f\right\|_{\infty}$. Choose $j_{0} \in \mathbb{N}$ according to (3.2). Since the functions $f 1_{|z|<R} * \phi_{n}$ converge to $f 1_{|x|<R} \in L^{1}(\mathbb{R})$ in the $L^{1}$ norm, a subsequence $f 1_{|z|<R} * \phi_{n_{k}}$ converges almost surely to $f 1_{|x|<R}$. For $n_{k} \geq j_{0}$,

$$
f 1_{|z|<R} * \phi_{n_{k}}(x)=f * \phi_{n_{k}}(x), \quad \text { if } \quad|x|<R-\delta .
$$

Thus, for almost all $|x|<R-\delta$,

$$
|f(x)|=\lim _{k \rightarrow \infty}\left|f * \phi_{n_{k}}\right| \leq M .
$$

Therefore, $|f(x)| \leq M$ for almost all $x \in \mathbb{R}$.

LEMMA 3.2. Assume that for some $s<t$ and all $x \in \mathbb{R}$,

$$
\int_{s}^{t} \int_{\mathbb{R}} p(s, x, u, z) q(z) \mathrm{d} z \mathrm{~d} u \leq M .
$$

Then, $q \in L_{\text {loc }}^{1}(\mathbb{R})$. 
Proof. Let $\varphi \in C_{0}(\mathbb{R})$ be such that $\varphi \geq 0, \varphi=1$ on $[0,1 / 2]$ and $\int_{\mathbb{R}} \varphi(x) d x=1$. For arbitrary fixed $x_{0} \in \mathbb{R}$, we have

$$
\begin{aligned}
M & \geq \int_{s}^{t} \int_{\mathbb{R}} \int_{\mathbb{R}} \varphi\left(x_{0}-x\right) p(s, x, u, z) d x q(z) \mathrm{d} z \mathrm{~d} u \\
& =\int_{s}^{t} \int_{\mathbb{R}} P_{u-s} \varphi\left(x_{0}-z\right) q(z) \mathrm{d} z \mathrm{~d} u \geq(\varepsilon / 2) \int_{x_{0}-1 / 2}^{x_{0}} q(z) \mathrm{d} z
\end{aligned}
$$

where $0<\varepsilon \leq t-s$ is such that $\left\|P_{u} \varphi-\varphi\right\|_{\infty} \leq 1 / 2$ for $u \leq \varepsilon$.

Lemma 3.2 is generalized to arbitrary Lévy processes in $\mathbb{R}^{d}[12$, Lemma 3.7].

THEOREM 3.3. Assume that for some $s<t$,

$$
\sup _{x<y} \int_{s}^{t} \int_{\mathbb{R}} \frac{p(s, x, u, z) p(u, z, t, y)}{p(s, x, t, y)} q(z) \mathrm{d} z \mathrm{~d} u<\infty .
$$

Then, $q \in L^{\infty}(\mathbb{R})$.

Proof. By the assumption, there is $M^{\prime}>0$ such that for some fixed $s<t$,

$$
\int_{s}^{t} \int_{\mathbb{R}} \frac{p(s, x, u, z) p(u, z, t, y)}{p(s, x, t, y)} q(z) \mathrm{d} z \mathrm{~d} u \leq M^{\prime}, \quad x<y .
$$

By Lemma 3.2, $q \in L_{l o c}^{1}(\mathbb{R})$. For $s<t$ and $n \in \mathbb{N}$, we let

$$
\phi_{n}(z)=\frac{1}{t-s} \int_{s}^{t} \frac{p(s,-1 / n, u,-z) p(u,-z, t, 1 / n)}{p(s,-1 / n, t, 1 / n)} \mathrm{d} u, \quad|z|<1 / n,
$$

and $\phi_{n}(z)=0$ for $|z| \geq 1 / n$. Clearly, $\phi_{n}$ satisfies conditions (3.1) and (3.2). Furthermore, for all $x \in \mathbb{R}$,

$$
\phi_{n} * q(x)=\frac{1}{t-s} \int_{s}^{t} \int_{\mathbb{R}} \frac{p(s, x-1 / n, u, z) p(u, z, t, x+1 / n)}{p(s, x-1 / n, t, x+1 / n)} q(z) \mathrm{d} z \mathrm{~d} u .
$$

Thus, $\sup _{n \in \mathbb{N}}\left\|\phi_{n} * q\right\|_{\infty} \leq M^{\prime} /(t-s)=M<\infty$. Lemma 3.1 ends the proof.

COROLLARY 3.4. Let $q(u, z)=q(z)$. Then, $q$ satisfies (1.6) if and only if $\|q\|_{\infty}<$ $\infty$. If there are $s<t$ and $C<\infty$ such that $\tilde{p}(s, x, t, y) \leq C p(s, x, t, y)$ for all $x<y$, then $\|q\|_{\infty}<\infty$.

Corollary 3.4 shows that the methods of [4] cannot deliver estimates of Schrödinger perturbations of transition densities $p$ of subordinators by unbounded time-independent $q$. In contrast, we saw in Sect. 2 that the methods based on majorants $p^{*}$ and $4 \mathrm{G}$ inequality handle such situations.

If we allow $q$ to depend on time, the statements of the corollary are no longer valid. Indeed, let $q(u, z)=u_{+}^{-1 / 2}$, where $u_{+}=u \vee 0$. Then, for all $s<t$ and $x<y$ and transition densities $p$,

$$
\int_{s}^{t} \int_{X} p(s, x, u, z) q(u, z) p(u, z, t, y) \mathrm{d} z \mathrm{~d} u \leq 2\left(t_{+}-s_{+}\right)^{1 / 2} p(s, x, t, y) .
$$


We see that this unbounded $q$ yields (1.6) and (1.7) for every $p$.

The next example builds on the ideas proposed in [15, Example 4].

EXAMPLE. Consider the second term $p_{1}$ of the perturbation series (1.2) for $\tilde{p}$. Let

$$
\sup _{\substack{s \leq u \leq t \\ x \leq z \leq y}} q(u, z) \leq \eta /(t-s)
$$

for some $\eta \geq 0$ and for all $s<t, x<y$ such that $(s, x),(t, y) \in F:=\{(u, z)$ : $q(u, z)>0\}$. Then, we claim that for all $s<t$ and $x<y$,

$$
p_{1}(s, x, t, y) \leq \eta p(s, x, t, y) .
$$

For the proof, we consider a Borel nondecreasing function $\omega:[s, t] \rightarrow \mathbb{R}, s<t$, such that $\omega(s)=x<y=\omega(t)$, and let $T(\omega)=\{u: s \leq u \leq t,(u, \omega(u)) \in F\}$. If $T(\omega)$ is empty, then

$$
\int_{s}^{t} q(u, \omega(u)) \mathrm{d} u=0 \leq \eta .
$$

Otherwise, we consider $\sigma=\inf \{u: u \in T(\omega)\}$ and $\tau=\sup \{u: u \in T(\omega)\}$. There are $s_{n} \leq t_{n}$ such that $\left(s_{n}, \omega\left(s_{n}\right)\right),\left(t_{n}, \omega\left(t_{n}\right)\right) \in F, s_{n} \downarrow \sigma$ and $t_{n} \uparrow \tau$, hence

$$
\begin{aligned}
\int_{s}^{t} q(u, \omega(u)) \mathrm{d} u & =\int_{\sigma}^{\tau} q(u, \omega(u)) \mathrm{d} u=\lim _{n \rightarrow \infty} \int_{s_{n}}^{t_{n}} q(u, \omega(u)) \mathrm{d} u \\
& \leq \lim _{n \rightarrow \infty}\left(t_{n}-s_{n}\right) \sup _{\substack{s_{n} \leq u \leq t_{n} \\
\omega\left(s_{n}\right) \leq z \leq \omega\left(t_{n}\right)}} q(u, z) \leq \eta .
\end{aligned}
$$

Finally, let $\left\{Y_{u}\right\}_{u \geq 0}$ be the subordinator. Given $s<t, x<y$ we denote by $\left\{Z_{u}\right\}_{s \leq u \leq t}$ the bridge corresponding to $\left\{Y_{u}\right\}_{u \geq 0}$, which starts from $x$ at time $s$ and reaches $y$ at time $t$. Since the trajectories of $\left\{Z_{u}\right\}_{u \geq 0}$ are almost surely nondecreasing, we have for all $s<t, x<y$,

$$
p_{1}(s, x, t, y) / p(s, x, t, y)=\mathbb{E}_{s, x}^{t, y}\left[\int_{s}^{t} q\left(u, Z_{u}\right)\right] \mathrm{d} u \leq \mathbb{E}_{s, x}^{t, y}[\eta]=\eta,
$$

as claimed.

Typical applications are $q(u, z)=\eta z 1_{(0,1 / u)}(z)$, cf. [15, Example 4] and $q(u, z)=$ $\eta z^{2} 1_{F}(u, z)$, where $F=\bigcup_{n=1}^{\infty}(1 /(n+1), n) \times(n-1, n)$. Both functions tend to infinity when time goes to zero and the space variable grows correspondingly.

We next show that the estimate (3.3) cannot be improved.

EXAMPLE. We define $q(u, z)=\eta z 1_{(0,1 / u)}(z), \eta>0$. Let $\varepsilon<\eta$. We claim that there is no superadditive $Q$ such that

$$
p_{1}(s, x, t, y) \leq[\varepsilon+Q(s, t)] p(s, x, t, y)
$$


Indeed, by [5, Lemma 5.3], we may assume that $Q$ is regular superadditive. Thus, there is $t$ such that $[\varepsilon+Q(0, t)]<(\varepsilon+\eta) / 2$. On the other hand, for $x:=(1+\varepsilon / \eta) /(2 t)<$ $y:=1 / t$, we have

$$
\begin{aligned}
p_{1}(s, x, t, y) & =\int_{0}^{t} \int_{x}^{y} p(s, x, u, z) q(u, z) p(u, z, t, y) \mathrm{d} z \mathrm{~d} u \\
& \geq \eta x \int_{0}^{t} \int_{x}^{y} p(s, x, u, z) p(u, z, t, y) \mathrm{d} z \mathrm{~d} u \\
& \geq \eta x t p(s, x, t, y)=[(\eta+\varepsilon) / 2] p(s, x, t, y),
\end{aligned}
$$

which is a contradiction.

\section{Auxilary results}

In this section, we prove (1.16) and its analogues in the setting of general semigroup theory. We consider a Banach space $(Y,\|\cdot\|)$. Let $T=\left(T_{t}\right)_{t \geq 0}$ be a strongly continuous semigroup of linear operators on $Y$. Let $L$ be the corresponding infinitesimal generator with domain $D(L)[18, \mathrm{IX}]$.

THEOREM 4.1. Let $\xi: \mathbb{R} \rightarrow D(L)$ be such that

$$
\begin{aligned}
t & \mapsto \xi(t) \text { is differentiable in }(Y,\|\cdot\|), \\
t & \mapsto \xi^{\prime}(t) \text { is continuous in }(Y,\|\cdot\|), \\
t & \mapsto L \xi(t) \text { is continuous in }(Y,\|\cdot\|), \\
t & \mapsto \xi(t) \text { has compact support in } \mathbb{R} .
\end{aligned}
$$

Then

$$
\int_{s}^{\infty} T_{u-s}\left[\xi^{\prime}(u)+L \xi(u)\right] \mathrm{d} u=-\xi(s), \quad s \in \mathbb{R},
$$

where the integral is the Riemann integral of a Banach space valued function.

Theorem 4.1 applies, e.g., to $\xi(t)=f(t) \xi_{0}$ with $\xi_{0} \in D(L)$ and $f \in C_{c}^{1}(\mathbb{R})$. Theorem 4.1 follows from two auxiliary lemmas.

LEMMA 4.2. If $\xi$ satisfies (4.1), then $t \mapsto T_{t} \xi(t)$ is differentiable in $(Y,\|\cdot\|)$ and

$$
\frac{\mathrm{d}}{\mathrm{d} t} T_{t} \xi(t)=T_{t} \xi^{\prime}(t)+T_{t} L \xi(t), \quad t \geq 0
$$

For $t=0$, the derivative is understood as the right-hand derivative. The lemma is a version of the differentiation rule for products.

Proof of Lemma 4.2. Let $h \neq 0(h>0$ if $t=0)$ and $h \rightarrow 0$. Clearly,

$$
\begin{aligned}
& \frac{T_{t+h} \xi(t+h)-T_{t} \xi(t)}{h} \\
& \quad=T_{t+h} \xi^{\prime}(t)+T_{t+h}\left(\frac{\xi(t+h)-\xi(t)}{h}-\xi^{\prime}(t)\right)+\left(\frac{T_{t+h}-T_{t}}{h}\right) \xi(t) .
\end{aligned}
$$


For some $M, \omega \geq 0$, we have $\left\|T_{t}\right\| \leq M e^{\omega t}, t \geq 0$ [18]. The lemma follows:

$$
\left\|T_{t+h}\left(\frac{\xi(t+h)-\xi(t)}{h}-\xi^{\prime}(t)\right)\right\| \leq M e^{\omega(t+h)}\left\|\frac{\xi(t+h)-\xi(t)}{h}-\xi^{\prime}(t)\right\| \rightarrow 0 .
$$

Let $a, b \in \mathbb{R}, a<b$. We write $\xi \in C^{1}([a, b], Y)$ if $\xi:[a, b] \rightarrow Y$ and (4.1) and (4.2) hold, with one-sided derivatives at the endpoints $a$ and $b$. Here is the fundamental theorem of calculus for Riemann-type Banach space integrals (see [9, Lemma 1.1.4] or [14, Lemma 2.3.24]).

LEMMA 4.3. If $\psi \in C^{1}([a, b], Y)$, then $\int_{a}^{b} \frac{\mathrm{d}}{\mathrm{d} u}[\psi(u)] \mathrm{d} u=\psi(b)-\psi(a)$.

Proof of Theorem 4.1. Let $s \in \mathbb{R}$. By Lemma 4.2, assumptions (4.2), (4.3) and (4.4), and by Lemma 4.3, we obtain the result:

$$
\int_{0}^{\infty} T_{u}\left[\xi^{\prime}(u+s)+L \xi(u+s)\right] \mathrm{d} u=\int_{0}^{\infty} \frac{\mathrm{d}}{\mathrm{d} u}\left[T_{u} \xi(u+s)\right] \mathrm{d} u=-\xi(s) .
$$

In fact, if $s$ is fixed, the assumptions on $\xi(t)$ only need to hold in $[s, \infty)$.

We shall give a partial converse to Theorem 4.1 by showing that the infinitesimal generator of $T$ is the only operator $L$ that makes (4.5) true.

THEOREM 4.4. Let $A$ be a linear operator on a linear space $D(A) \subset Y$ with values in $Y$. Assume that $\xi: \mathbb{R} \rightarrow D(A)$ is such that

$$
\begin{aligned}
& t \mapsto \xi(t) \text { is differentiable in }(Y,\|\cdot\|), \\
& t \mapsto \xi^{\prime}(t) \text { is continuous in }(Y,\|\cdot\|), \\
& t \mapsto A \xi(t) \text { is continuous in }(Y,\|\cdot\|), \\
& t \mapsto \xi(t) \text { has compact support in } \mathbb{R}, \\
& \int_{s}^{\infty} T_{u-s}\left[\xi^{\prime}(u)+A \xi(u)\right] \mathrm{d} u=-\xi(s), \quad s \in \mathbb{R} .
\end{aligned}
$$

Then $\xi(t) \in D(L)$ and $L \xi(t)=A \xi(t)$ for all $t \in \mathbb{R}$.

Proof. Let $t \in \mathbb{R}$ and $h>0$. By (4.11),

$$
\begin{aligned}
\int_{t+h}^{\infty} T_{u-t}\left[\xi^{\prime}(u)+A \xi(u)\right] \mathrm{d} u & =\int_{t+h}^{\infty} T_{u-(t+h)} T_{h}\left[\xi^{\prime}(u)+A \xi(u)\right] \mathrm{d} u \\
& =-T_{h} \xi(t+h) .
\end{aligned}
$$

Subtracting this from (4.11) with $s=t$, we get

$$
\int_{t}^{t+h} T_{u-t}\left[\xi^{\prime}(u)+A \xi(u)\right] \mathrm{d} u=T_{h} \xi(t+h)-\xi(t) .
$$


We get

$$
\left(\frac{T_{h}-I}{h}\right) \xi(t)=\frac{1}{h} \int_{t}^{t+h} T_{u-t}\left[\xi^{\prime}(u)+A \xi(u)\right] \mathrm{d} u-T_{h}\left(\frac{\xi(t+h)-\xi(t)}{h}\right) .
$$

By (4.7)-(4.9), the limit on the right-hand side exists as $h \rightarrow 0^{+}$and equals

$$
L \xi(t)=T_{0}\left(\xi^{\prime}(t)+A \xi(t)\right)-T_{0} \xi^{\prime}(t)=A \xi(t) .
$$

In fact, the assumptions (4.7)-(4.11) only need to hold on $[t, t+\varepsilon), \varepsilon>0$.

REMARK 4.5. We call $\xi$ satisfying (4.7)-(4.10) a path for A. Define

$$
D(A, T)=\{\xi(t): \text { such that } t \in \mathbb{R} \text {, and } \xi \text { is a path for } A \text { satisfying (4.11) }\}
$$

If $A$ is the infinitesimal generator of a strongly continuous semigroup $S=\left(S_{t}\right)_{t \geq 0}$ on $Y$ and $D(A, T)$ contains the cores of $L$ and $A$, then $L \equiv A$ and $T \equiv S$. Indeed, by the comment following Theorem 4.1, for the infinitesimal generator $L$ of $T=\left(T_{t}\right)_{t \geq 0}$, we have $D(L, T)=D(L)$. Theorem 4.4 means that $D(A, T) \subseteq D(A) \cap D(L)$, and $A=L$ on $D(A, T)$. This identifies $L$ with $A$ and $T$ with $S$.

We now focus on Lévy semigroups discussed in the Introduction.

Proof of (1.16). Recall that $C_{c}^{\infty}\left(\mathbb{R}^{d}\right) \subset C_{0}^{2}\left(\mathbb{R}^{d}\right) \subset D(L)$. We shall verify the assumptions of Theorem 4.1 for $\xi(t)=\phi(t, \cdot)$. It suffices to justify (4.3). Recall that (1.15) holds for $f \in C_{0}^{2}\left(\mathbb{R}^{d}\right)$ and $L$ is continuous from $C_{0}^{2}\left(\mathbb{R}^{d}\right)$ to $C_{0}\left(\mathbb{R}^{d}\right)$ [17, p. 211]. We note that $t \mapsto \phi(t, \cdot)$ is continuous in $C_{0}^{2}\left(\mathbb{R}^{d}\right)$. Therefore, $t \mapsto L \phi(t, \cdot)$ is continuous in $\left(C_{0}\left(\mathbb{R}^{d}\right),\|\cdot\|_{\infty}\right)$. By Theorem 4.1 ,

$$
-\xi(s)=\int_{s}^{\infty} P_{u-s}\left[\xi^{\prime}(u)+L \xi(u)\right] \mathrm{d} u
$$

in $C_{0}\left(\mathbb{R}^{d}\right)$. Recall that the Riemann integrals converge in norm. Evaluation at a point is continuous on $\left(C_{0}\left(\mathbb{R}^{d}\right),\|\cdot\|_{\infty}\right)$; therefore, the above identity holds pointwise, i.e., (1.16) holds. We note in passing that the integral in (1.16) may be interpreted as Lebesgue integral on $\mathbb{R} \times \mathbb{R}^{d}$.

THEOREM 4.6. (Uniqueness). Let $C_{c}^{\infty}\left(\mathbb{R}^{d}\right)$ be a core of a closed linear operator $A$ with domain $D(A) \subset\left(C_{0}\left(\mathbb{R}^{d}\right),\|\cdot\|_{\infty}\right)$. If for all $s \in \mathbb{R}, x \in \mathbb{R}^{d}$ and $\phi \in C_{c}^{\infty}\left(\mathbb{R} \times \mathbb{R}^{d}\right)$,

$$
\int_{s}^{\infty} \int_{\mathbb{R}^{d}}\left[\partial_{u} \phi(u, x+z)+A \phi(u, x+z)\right] p_{u-s}(\mathrm{~d} z) \mathrm{d} u=-\phi(s, x),
$$

then $A \equiv L$.

Proof. For $\varphi \in C_{c}^{\infty}\left(\mathbb{R}^{d}\right)$ and $f \in C_{c}^{1}\left(\mathbb{R}^{d}\right)$, we let $\xi(t)=f(t) \varphi$. Then, $\xi$ is a path for $A$ and $\zeta(t):=\int_{t}^{\infty} P_{u-t}\left[\xi^{\prime}(u)+A \xi(u)\right] \mathrm{d} u \in C_{0}\left(\mathbb{R}^{d}\right)$ converges in norm. By continuity of evaluations and (4.12) with $\phi(t, x)=f(t) \varphi(x)$, we have $\zeta(t)(x)=-\xi(t)(x)$, $t \in \mathbb{R}, x \in \mathbb{R}^{d}$. By Theorem $4.4, A=L$ on the common core $C_{c}^{\infty}\left(\mathbb{R}^{d}\right)$. This ends the proof. 
REMARK 4.7. If the Lévy process $\left\{X_{t}\right\}$ has a (transition) density function, i.e., $p_{t}(\mathrm{~d} y)=p(t, y) \mathrm{d} y$ for $t>0$, then (1.16) reads as

$$
\int_{s}^{\infty} \int_{\mathbb{R}^{d}} p(u-s, z-x)\left[\partial_{u} \phi(u, z)+L \phi(u, z)\right] \mathrm{d} z \mathrm{~d} u=-\phi(s, x) .
$$

We shall focus on the case when $d=1$ and $\left\{X_{t}\right\}$ is a subordinator, i.e., a nondecreasing Lévy process. The Lévy measure $v$ of $X_{t}$ is concentrated on $(0, \infty)$. Since $\int(x \wedge 1) v(d x)<\infty$ and $L$ is a closed operator, (1.15) may be rearranged: We obtain $C_{0}^{1}(\mathbb{R}) \subset D(L)$ and

$$
L f(x)=b \frac{d f}{d x}(x)+\int_{0}^{\infty}(f(x+y)-f(x)) v(\mathrm{~d} y), \quad f \in C_{0}^{1}(\mathbb{R}) .
$$

Here $b \geq 0$ is the drift coefficient. Furthermore, for $f \in C_{c}^{1}(\mathbb{R})$ we obtain

$$
\begin{aligned}
\int_{0}^{\infty}(f(x+y)-f(x)) v(\mathrm{~d} y) & =\int_{0}^{\infty} \int_{0}^{y} f^{\prime}(x+z) \mathrm{d} z v(\mathrm{~d} y) \\
& =\int_{0}^{\infty} f^{\prime}(x+z)\left(\int_{z}^{\infty} v(\mathrm{~d} y)\right) \mathrm{d} z .
\end{aligned}
$$

Let $\bar{v}(z)=\int_{z}^{\infty} v(\mathrm{~d} y)$. We thus have

$$
L f(x)=b f^{\prime}(x)+\int_{x}^{\infty} f^{\prime}(z) \bar{v}(z-x) \mathrm{d} z, \quad f \in C_{c}^{1}(\mathbb{R}) .
$$

EXAMPLE. Let $\alpha \in(0,1)$ and $\left\{X_{t}\right\}$ be the $\alpha$-stable subordinator, i.e.,

$$
b=0 \quad \text { and } \quad v(\mathrm{~d} y)=\frac{\alpha}{\Gamma(1-\alpha)} y^{-\alpha-1} 1_{y>0} \mathrm{~d} y .
$$

We then see that the generator of $\left\{X_{t}\right\}$ coincides on $C_{c}^{1}(\mathbb{R})$ with the Weyl fractional derivative (cf. (1.17) for the case $\alpha=1 / 2$ ). The potential operator for $\left\{X_{t}\right\}$ is the Weyl fractional integral

$$
W^{-\alpha} f(x)=\int_{0}^{\infty} T_{t} f(x) \mathrm{d} t=\frac{1}{\Gamma(\alpha)} \int_{x}^{\infty} f(z)(z-x)^{\alpha-1} \mathrm{~d} z, \quad f \in C_{c}(\mathbb{R}) .
$$

We note in passing that $-W^{-\alpha} \partial^{\alpha}=I$ (the identity operator) on $C_{c}^{1}(\mathbb{R})$. Schrödinger perturbations of $W^{-\alpha}$ were discussed in [4, Example 2 and 3]. The discussion was facilitated by the fact that the $3 \mathrm{G}$ Theorem holds for $(y-x)_{+}^{\alpha-1} / \Gamma(\alpha)$.

EXAMPLE. Since the inverse Gaussian subordinator is obtained by the Esscher transform (tempering) and time rescaling of the 1/2-stable subordinator (cf. [7], Sec. 4.4.2), for $f \in C_{c}^{1}(\mathbb{R})$, the generator of the inverse Gaussian subordinator is given by (1.20).

REMARK 4.8. For (signed) $q: \mathbb{R} \times X \rightarrow \mathbb{R}$, we define the Schrödinger perturbation $\tilde{p}$ of $p$ by $q$ by exactly the same formulas (1.2) and (1.3). We get (1.4), (1.5), ChapmanKolmogorov, provided the perturbation series for $|q|$, which gives an upper bound for $\tilde{p}$, is finite. Under this condition, Lemma 2.1 remains valid, too. For lower bounds of $\tilde{p}$ for signed $q$, we refer to $[2,5]$. 


\section{Acknowledgements}

We thank Tomasz Jakubowski and Sebastian Sydor for discussions and Zhen-Qing Chen for a question motivating Theorem 4.4. We thank the referees for very helpful suggestions, which considerably improved the paper. Krzysztof Bogdan was partially supported by NCN Grant 2012/07/B/ST1/03356. Karol Szczypkowski was partially supported by NCN Grant 2011/03/N/ST1/00607.

Open Access. This article is distributed under the terms of the Creative Commons Attribution 4.0 International License (http://creativecommons.org/licenses/by/4.0/), which permits unrestricted use, distribution, and reproduction in any medium, provided you give appropriate credit to the original author(s) and the source, provide a link to the Creative Commons license, and indicate if changes were made.

\section{REFERENCES}

[1] Applebaum, D. Lévy processes and stochastic calculus, 2 ed., vol. 116 of Cambridge Studies in Advanced Mathematics. Cambridge University Press, Cambridge, 2009.

[2] Bogdan, K., Hansen, W., And Jakubowski, T. Time-dependent Schrödinger perturbations of transition densities. Studia Math. 189, 3 (2008), 235-254.

[3] Bogdan, K., Hansen, W., And Jakubowski, T. Localization and Schrödinger perturbations of kernels. Potential Anal. 39, 1 (2013), 13-28.

[4] Bogdan, K., JAKubowski, T., AND Sydor, S. Estimates of perturbation series for kernels. $J$. Evol. Equ. 12, 4 (2012), 973-984.

[5] BogdAN, K., AND SzCZYPKOWSKI, K. Gaussian estimates for Schrödinger perturbations. Studia Math. 221, 2 (2014), 151-173.

[6] ButKo, YA. A. On fundamental solutions, transition probabilities and fractional derivatives. Science and Education of the Bauman MSTU, 1 : 42-52, 2015. DOI:10.7463/0115.0754986

[7] CONT, R., AND TANKOV, P. Financial modelling with jump processes. Chapman \& Hall/CRC Financial Mathematics Series. Chapman \& Hall/CRC, Boca Raton, FL.

[8] Dynkin, E. B. Diffusions, superdiffusions and partial differential equations, vol. 50 of American Mathematical Society Colloquium Publications. American Mathematical Society, Providence, RI, 2002.

[9] EThIER, S. N., AND KuRTZ, T. G. Markov processes. Wiley Series in Probability and Mathematical Statistics: Probability and Mathematical Statistics. John Wiley \& Sons Inc., New York, 1986. Characterization and convergence.

[10] Frazier, M., NAZARov, F., AND Verbitsky, I. Global estimates for kernels of Neumann series and Green's functions. J. London Math. Soc. 90, 3 (2014), 903-918.

[11] GRIGOR'YAN, A., AND HANSEN, W. Lower estimates for a perturbed Green function. J. Anal. Math. 104 (2008), 25-58.

[12] GRZYwny, T., AND SzCZYPKOWsKi, K. Kato classes for Lévy processes. ArXiv e-prints (Mar. 2015).

[13] Hansen, W. Global comparison of perturbed Green functions. Math. Ann. 334, 3 (2006), $643-678$.

[14] JACOB, N. Pseudo differential operators and Markov processes. Vol. I. Imperial College Press, London, 2001. Fourier analysis and semigroups.

[15] JAKubowski, T. On combinatorics of Schrödinger perturbations. Potential Anal. 31,1 (2009), 45-55.

[16] Räbiger, F., Schnaubelt, R., Rhandi, A., And Voigt, J. Non-autonomous Miyadera perturbations. Differential Integral Equations 13, 1-3 (2000), 341-368.

[17] SATO, K.- I. Lévy processes and infinitely divisible distributions, vol. 68 of Cambridge Studies in Advanced Mathematics. Cambridge University Press, Cambridge, 1999. Translated from the 1990 Japanese original, Revised by the author.

[18] YosidA, K. Functional analysis, sixth ed., vol. 123 of Grundlehren der Mathematischen Wissenschaften [Fundamental Principles of Mathematical Sciences]. Springer-Verlag, Berlin, 1980. 
[19] ZHANG, Q. S. On a parabolic equation with a singular lower order term. II. The Gaussian bounds. Indiana Univ. Math. J. 46, 3 (1997), 989-1020.

[20] ZHANG, Q. S. A sharp comparison result concerning Schrödinger heat kernels. Bull. London Math. Soc. 35, 4 (2003), 461-472.

K. Bogdan and K. Szczypkowski

Wroctaw University of Technology,

Wybrzeże Wyspiańskiego 27,

50-370 Wroctaw, Poland

E-mail:bogdan@pwr.edu.pl

K. Szczypkowski

E-mail: karol.szczypkowski@math.uni-bielefeld.de, karol.szczypkowski@pwr.edu.pl

Y. Butko

Bauman Moscow State Technical University, 2nd Baumanskaya Str. 5, Moscow, 105005 Russia E-mail: yanabutko@yandex.ru,

kinderknecht@math.uni-sb.de

and

University of Saarland,

P.O. Box 1511 50,

66041 Saarbrücken, Germany

K. Szczypkowski

Universität Bielefeld,

Postfach 1001 31,

33501 Bielefeld, Germany 